\title{
Platform-Independent Benchmarks for Task and Motion Planning
}

\author{
Fabien Lagriffoul* Neil T. Dantam ${ }^{\circledR}$ Caelan Garrett $^{\dagger}$ Aliakbar Akbari $^{\S}$ Siddharth Srivastava $^{\ddagger}$ Lydia E. Kavraki $^{ \pm}$
}

\begin{abstract}
We present the first platform-independent evaluation method for Task and Motion Planning (TAMP). Previously point, various problems have been used to test individual planners for specific aspects of TAMP. However, no common set of metrics, formats, and problems have been accepted by the community. We propose a set of benchmark problems covering the challenging aspects of TAMP and a planner-independent specification format for these problems. Our objective is to better evaluate and compare TAMP planners, foster communication and progress within the field, and lay a foundation to better understand this class of planning problems.
\end{abstract}

Index Terms-Performance Evaluation and Benchmarking, Task Planning, Manipulation Planning

\section{INTRODUCTION}

Everyday activities require reasoning about both high-level task actions and specific geometric motions. Systems that perform both task planning and motion planning have existed for a long time, e.g., the pioneering robot Shakey [1]. These systems were traditionally rooted in the classical sense-planact paradigm, which separated the planning of logical task actions from the execution of specific motions. However, the increasing complexity of robots and their capacity to manipulate the environment has uncovered a new class of problems, which cannot be solved by considering task actions and geometric motions in isolation. Many activities feature dependencies between logical and geometrical levels. Deciding which actions to do-and in which order-is closely linked to how these actions can be physically performed. Thus, it requires planning techniques that combine both task planning and motion planning.

Combined task and motion planning is an active research area with work proceeding in the robotics, AI, and formal methods communities. Though the broad aims of these works are similar, the focus spans many related areas: planar navigation [2], rearranging objects [3], dynamics in manipulation [4], humanoid robots [5], achieving optimality [6]. The variation in focus, assumptions, and scenarios presents challenges when attempting to directly compare planners and measure the forward progress of the field. This paper presents a set of criteria, metrics, and scenarios for the evaluation, comparison, and benchmarking of Task-Motion planners.

\footnotetext{
* Örebro University, Sweden. Fabien.Lagriffouleoru. se

$\dagger$ Massachusetts Institute of Technology, USA. caelan@mit.edu

$\S$ Univ. Politèc de Catalunya, Spain. aliakbar.akbari@upc.edu

¥ Arizona State University, USA. siddharths@asu.edu

- Colorado School of Mines, USA. ndantamemines .edu

\pm Rice University, USA. kavraki@rice.edu
}

The evaluation approach in this paper is a collaborative development following TAMP workshops at RSS 2016 and 2017. We have produced a common set of benchmarks ${ }^{1}$ that are independent of any of our specific planning systems. Beyond the initial comparison of planners, we believe that a common set of benchmarks - and ultimately a planning competition-is crucial to promote development and measure research progress, as demonstrated by the success of the International Planning Competition (IPC) [7], the Satisfiability Modulo Theories Competition (SMT-COMP) [8], or robotics competitions such as RoboCup [9] and RoCKIn [10]. Through the present work, we anticipate that other researchers will join our initiative to improve and refine this evaluation method or even develop a superior successor. The fundamental prerequisite for this benchmarking approaches is community consensus on the choice of benchmark problems and methods. This paper is a first step in this direction.

The paper is organized as follows: section II presents prior work on TAMP. section III gives an overview of TAMP and discusses the assumptions made in this paper. Based on these assumptions, we formally define TAMP as considered for this work in section IV. section $V$ explains the data and specification formats we adopt for TAMP problems. section VI introduces the benchmarks problems and discusses how they were developed. section VII offers practical details about using the benchmark data and evaluation metrics. Finally, section VIII provides a summary and perspective on future work.

\section{PRIOR WORK ON TAMP}

The first work combining logical and geometric search spaces originates from both the robotics and AI communities. Cambon et al. developed aSyMov [11] in 2004, a motion planner for multiple robots using AI planning as a heuristic for probabilistic roadmap search. Likewise in the AI planning community, Dornhege et al. introduce semantic attachments in 2009 in order to account for the geometric side effects of logical actions [12]. Navigation Among Movable Obstacles (NAMO) [13] relates to TAMP in that it combines motion planning together with discrete choices about obstacles to move. However, NAMO focuses on a specific type of discrete action (moving obstacles) in contrast to general task planning in TAMP.

TAMP has become an active field of research in both AI and Robotics communities, reflected by the emergence of dedicated workshops, conference tracks, and journal special

${ }^{1}$ http://tampbenchmark.aass.oru.se 
issues: AAAI 2010 (workshop on Bridging the gap between Task and Motion Planning), ICAPS (PlanRob workshop since 2012, Robotic Track since 2015), RSS since 2013 (workshop on Task and Motion Planning), IROS 2013-2015 (AI-based Robotics, AI and Robotics, Task Planning for Intelligent Robots), AI Journal 2014 (special issue on AI and Robotics), and the International Journal of Robotics Research's current call for a special issue on Task and Motion Planning.

Planning systems combining both types of planning have flourished [14], [15], [16], [17], [18], [19], [20], [21], [4], [22], [23], together with their own planner-specific evaluations, yet no common benchmarks have so far been established. These systems are evaluated on subsets of possible problems which do not cover all the features of TAMP, therefore leading to (over)specialized planners [24]. The topic of benchmarks for TAMP is recurrently proposed in dedicated workshops, and we hope through this paper to finally address the issue.

\section{OVERVIEW OF TAMP}

TAMP combines logical and geometric reasoning. Robots need logical reasoning to determine which actions are needed to achieve a given goal, and they need geometric reasoning to know if and how these actions can be physically performed. TAMP addresses this issue by combining discrete task decisions about objects and actions with continuous motion decisions about paths.

Typical algorithms for independent task planning and motion planning are fundamentally different. Task planning finds a discrete sequence of actions to transition from a given start state to a desired goal condition, typically using heuristic search or constraint satisfaction. Geometric motion planning finds a collision-free path from a given start configuration to a desired goal, typically using sampling or optimization-based methods. TAMP combines these two planning domains and addresses the interaction between them.

The range of problems which may be considered "TAMP" is large, spanning geometric vs. dynamic, fully vs. partially observable, deterministic vs. non-deterministic, and singleagent vs. adversarial vs. collaborative cases. For the scope of this work, we have necessarily restricted the focus to produce a workable benchmark set. We hope that further evaluation and developments will lead to standardized benchmarks for broader problem classes in the future.

\section{A. Assumptions}

This benchmark set addresses the geometric, fullyobservable, deterministic, single-agent subset of TAMP. We focus on the computational complexity of searching coupled logical and geometric spaces, in contrast to other areas of robotics which may primarily focus on uncertainty and partial observability. Hence, we make the following assumptions:

- Geometric: Motion planning over positions only is sufficient. Objects that are grasped or placed are kinematically coupled with the parent object and do not move or slide.

- Fully Observable: The initial state is entirely known geometrically (positions, meshes, configurations), semantically (e.g., movability of objects, placement locations).
- Deterministic: State is only changed by the planned actions. Robot motions and object grasp/release operations exactly follow the output of the path planner.

\section{B. Specification of TAMP Problems}

1) Task Requirements: At a high level, task planners search through a discrete transition system (see 1). We need a specification format that is sufficiently expressive and compact to represent our domains of interest. The Planning Domain Definition Language (PDDL) [25], [26] is a task modeling language with wide support due to its use in the International Planning Competition [7]. PDDL's status as a de facto standard makes it a natural choice to exchange task domains for this benchmark set. Though the problems are specified in PDDL (see subsection V-A), we anticipate that task-motion planners will also use other representations internally, e.g., answer set programming (ASP) [27], SMTLib [28], or temporal logics, based on specific implementation decisions. We discuss the details of the task specification format in subsection V-A.

2) Motion Requirements: Abstractly, motion planners find paths through a (typically continuous) configuration space. Thus, we need to specify the configuration space for the motion planner. For this initial benchmark set, we focus on geometric motion planning and leave dynamic or physicsbased planning [4], [29], [30] to future benchmark sets. The geometric case is sufficient for many manipulation scenarios. The configuration space for the motion planner is based on the robot's kinematics, i.e., joints, and the positions of the rigid bodies in the environment. There are many equivalent formats to specify robot kinematics and rigid body geometry. We discuss the details of the motion specification format in subsection V-B.

\section{TAMP PROBLEM DEFINITION}

We formulate the TAMP problem starting from the conventional formulations of task planning [31] and motion planning [32], [33].

\section{A. Task Planning}

Task planning finds a sequence of discrete or symbolic actions from an initial state to a desired goal state. While task planning covers a wide range of problems, all notations and representations correspond to a state-transition-system [34]:

Definition 1 (Task State-Transition-System): A task domain is the tuple $\Sigma=\left(S, A, \gamma, s_{0}, S_{g}\right)$ where,

- $S$ is a finite set of states

- $A$ is a finite set of actions

- $\gamma: S \times A \rightarrow S$ is a deterministic state-transition function, thus gives one state when applicable, which we denote by $\gamma(s, a)=s^{\prime}$.

- $s_{0} \in S$ is the start state

- $S_{g} \subseteq S$ is the set of goal states

Definition 2 (Task Plan): A task plan for domain $\Sigma=$ $\left(S, A, \gamma, s_{0}, g\right)$ is the sequence $\left\langle a_{0}, a_{1}, \ldots, a_{n}\right\rangle$, where each $a_{i} \in A$, for $0 \leq i<n, s_{i+1}=\gamma\left(s_{i}, a_{i}\right)$, and $s_{n} \in g$. That is, the task plan is a string in the language of $\Sigma$. 
Since for even small task planning problems, it is computationally infeasible to explicitly represent all states $S$, one must instead use various compact, symbolic representations, e.g., the Planning Domain Definition Language (PDDL) [25], Linear Temporal Logic (LTL) [35], Answer Set Programming (ASP) [27], $\mathcal{C}+[36]$, etc.

\section{B. Motion Planning}

Abstractly, motion planning finds valid paths through a configuration space $\mathcal{C}$ from a given start to a given goal state. The free configuration space, $\mathcal{C}_{\text {free }} \subseteq \mathcal{C}$, is the space of configurations, e.g., configurations where the robot does not collide with objects or itself.

Definition 3 (Motion Plan): Given free configuration space $\mathcal{C}_{\text {free }}$, initial configuration $q_{I} \in \mathcal{C}_{\text {free }}$, and goal configurations $G \subseteq \mathcal{C}_{\text {free }}$, a motion plan is defined as either:

1) A sequence $Q=\left\langle q_{0}, \ldots, q_{n}\right\rangle$ where $q_{0}=q_{I}$ is the start configuration, $q_{n} \in G$ is a goal configuration, each $q_{i} \in$ $\mathcal{C}_{\text {free }}$ is valid, and subsequent configurations are nearby $\left\|q_{i+1}-q_{i}\right\| \leq \epsilon$

2) Or a continuous trajectory $\tau:[0,1] \rightarrow \mathcal{C}_{\text {free }}$ such that $\tau(0)=q_{I}$ and $\tau(1) \in G$.

Typically, we will conduct motion planing in planar or 3dimensional worlds- $\mathcal{W}=\mathbb{R}^{2}$ or $\mathcal{W}=\mathbb{R}^{3}$-with obstacle region $\mathcal{O}$, and one or more multi-jointed robots. A configuration $q \in \mathcal{C}$ corresponds to joint position vector for the robot, and a free configuration $q \in \mathcal{C}_{\text {free }}$ is a joint position vector not in collision. Widely-used software packages model the configuration space of robot manipulators using kinematic trees or scene graphs [37], [38], [39] of local coordinate frames and attached rigid-body geometry (i.e., meshes), connected by the robots' joints. Explicit representations of the free configuration space $\mathcal{C}_{\text {free }}$ are generally infeasible to produce, so we instead use "blackbox" collision checkers [40] to test the validity (freedom-from-collision) of configurations during planning.

\section{Task-Motion Planning}

The interaction between task planning and motion planning is fundamental to TAMP. However, different planning approaches have formalized task-motion interaction differently. Thus, we first informally discuss the necessary relations between task planning and motion planning. Then, we summarize different specific formalizations. Finally, we present a minimal interaction specification for the benchmark set.

TAMP requires that we establish relationships between (1) states and configurations and (2) actions and motion plans. These relations ensure consistence between symbolic and geometric domains. Consistence implies, for instance, that the initial state $s_{0} \in S$ correspond to the initial configuration $q_{0} \in \mathcal{C}$. Similarly, each action $a \in A$ in the task plan can only occur if there exists a corresponding feasible motion plan $\tau_{a} \in \mathcal{C}_{\text {free. }}$ Actions that change the free configuration space-e.g., grasping an object with the robot's grippermust be reflected at the motion planning level, e.g., with an additional fixed joint between object and gripper. Finally, symbolic-geometric consistence implies that during execution of a motion plan, the world does not undergo uncontrolled state transitions, i.e., given the state transition $\gamma(s, a)=s^{\prime}$, the configurations traversed by the motion plan cannot be mapped to a state other than $s$ or $s^{\prime}$. Based on these necessary relations, we now summarize different specific approaches to task-motion interaction.

Various methods and formalizations of task-motion interaction are used by different planning systems. The domain semantics approach introduces a pair of functions to define taskmotion interaction in terms of abstraction and refinement [41], [42]. The abstraction function maps from a scene graph to a task state, and the refinement function maps from an action to a motion planning goal and an updated scene graph. Similar to the aforementioned refinement, Semantic attachments [12] connect PDDL-type actions to external geometric procedures, and the Planner-Independent Interface Layer [19] implements a symbolic-to-geometric mapping. Pre-computing reachability maps is another approach to ensure the semantics of a task domain or a logic program to reflect geometric constraints [16], [43], [14]. The sample-based approach [44] introduces conditional samplers to generate configurations corresponding to task states or actions. For example, a conditional sampler for a grasp action would generate configurations based on inverse kinematics to put the manipulator in a grasping configuration.

For the scope of this paper, we adopt a simplified model of task-motion interactions with the aim of maximizing compatibility with various planning systems. We use two abstract mapping functions:

- $\phi: S \rightarrow 2^{C}$ which maps states to configurations;

- $\xi: A \rightarrow 2^{C}$ which maps actions to motion plans.

As an example, if task state $s$ indicates "the robot is in the kitchen", $\phi(s)$ encompasses all the configurations such that the robot is located in the kitchen, grasping or not all available objects in any possible ways, the remaining objects lying in all possible poses. $\phi$ and $\xi$ are further described in subsection $\mathrm{V}-\mathrm{C}$ when we present a concrete implementation of task-motion interactions in the context of manipulation problems.

Based on this minimal specification of task-motion interaction, we now define the TAMP problem.

Definition 4 (Task-Motion Planning): Given a tuple $\left(\mathcal{C}, \Sigma, \phi, \xi, q_{0}\right)$, the TAMP problem is to find a sequence of actions $\left\langle a_{0}, a_{1}, \ldots, a_{n-1}\right\rangle$ following a sequence of task states $\left\langle s_{0}, s_{1}, \ldots, s_{n}\right\rangle$ such that

$$
\begin{aligned}
& s_{n} \in S_{g} \text { and } \\
& s_{i+1}=\gamma\left(s_{i}, a_{i}\right)
\end{aligned}
$$

and to find a sequence of motion plans $\left\langle\tau_{0}, \tau_{1}, \ldots, \tau_{n-1}\right\rangle$ such that $\forall i=0 \ldots n-1$ :

$$
\begin{aligned}
& \tau_{i}(0) \in \phi\left(s_{i}\right) \text { and } \tau_{i}(1) \in \phi\left(s_{i+1}\right) \\
& \tau_{i} \in \xi\left(a_{i}\right), \text { and } \\
& \tau_{i}(1)=\tau_{i+1}(0)
\end{aligned}
$$




\section{Specification of Benchmark Problems}

The data and formats to specify benchmark problems are a necessary part of any planner-independent test suite for TAMP. Thanks to existing benchmarks, competitions [7], and software packages [39], there are already many standard formats and languages for task planning and motion planning in isolation. Thus, we adopt the standard and conventional formats for the task domain (see subsection IV-A) and the motion domain (subsection IV-B). For task-motion interaction ( subsection IV-C) at the current stage, we provide high-level semantic information on grasp points, placement locations, etc. with the hope of stimulating further testing and development leading to an eventual standard format.

\section{A. Task Specification}

The de facto standard format for task domains is the Planning Domain Definition Language (PDDL) [26] developed for the International Planning Competition [7]. PDDL defines the task language in terms of actions with symbolic (Boolean expression) preconditions and effects, an initial state, and a symbolic expression for the set of goal states. A transition in the task language corresponds to taking a PDDL action, where the PDDL precondition holds in the predecessor state and the PDDL effect sets the successor state. PDDL compactly represents large state spaces using symbolic expressions for sets of states. Figure 1 shows an example PDDL specification for the classic Towers of Hanoi problem, detailed in subsection VI-A.

While we adopt PDDL for the benchmark set as a standard interchange format for transitions sytems, this choice does not preclude use of other representations, e.g., ASP, LTL, within planners. The choice of modeling language may, however, influence the focus of planners [45], and we hope further use of the benchmarks will yield refinements to the task specification format.

\section{B. Motion Specification}

The essentially universal model for robot manipulators is the kinematic tree or scene graph with attached rigid body geometry (meshes) [37], [38], [39], [42]. However, there are various alternative formats to specify the tree structure and the mesh data. We briefly summarize some major formats and explain the choices for the benchmark set aimed at maximizing compatibility.

1) Kinematics: Historically, Denavit-Hartenberg (DH) parameters [46] were a common approach to specify manipulator kinematics, though they did not address the meshes necessary for collision detection. The ROS Universal Robot Definition Format (URDF) [47] is a currently popular representation for robot manipulators with widespread support from robot vendors and the ability to reference external mesh files. Due to the wide availability of URDF models for many robots, we specify the benchmark problems using URDF.

2) Geometry: Numerous formats for mesh data have been developed by the animation and computer-aided design (CAD) communities. One attempt at a universal interchange format is the XML-based COLLADA [48]. However, the COLLADA specification is difficult to implement and software support and compatibility is poor. The Wavefront Object (OBJ) format is a lightweight representation for mesh data that is humanreadable and easy to parse. Wavefront OBJ is widely supported among animation and CAD software packages. Thus, we specify meshes for the benchmark set using OBJ.

\section{Specification of Task-Motion Interaction}

TAMP planners use their own symbolic-geometric mappings, hence there is currently no standard language to specify task-motion interaction. This stems from the fact that (1) at the task level, domains can be modeled at arbitrary levels of abstraction, and (2) at the motion level, there are various ways of implementing motion primitives. Consider for instance a planner using the following motion primitive for grasping: "reach $X$ with the manipulator, close the gripper, lift $X$ off the table by $5 \mathrm{~cm}$ ", while another planner uses: "reach $X$ with full-body motion, close the gripper". Comparing both planners would not be fair because they do not afford the same possibilities of action in the first place, therefore they cannot reach the same solution space.

To promote fair comparison of planners, we provide simplified symbolic-geometric mappings $\phi$ and $\xi$ (see subsection IV-C) for manipulation scenarios. This semantic information is based on both the properties of the robot and environment. The existing Semantic Robot Definition Format (SRDF) provides some of necessary information but does not fully cover the needs of Task-Motion interaction. We provide the data relating task actions and motions as XML files ${ }^{2}$ included with the benchmarks.

1) State-configuration mapping $(\phi)$ : We make the following assumptions which are restrictive, but allow us to specify $\phi$ unambiguously and make sure that different planners actually address the same problems:

- Surfaces Supporting Stable Placement (SSSP): When moved, objects can only be placed at these regions. An SSSP is a flat polygon, segment, or point attached to an object (see Figure 2).

- Stable Object Poses (SOP) : At rest, objects have to lie in one of these predefined poses. An SOP is represented by a rotation matrix, an axis of rotation (both in world frame) and a distance (to a SSSP) (see Figure 3).

For each object, an optional set of grasps is provided, i.e., the planner may use it or not. A discrete grasp is represented by a grasp frame (in the reference frame of the object), and a continuum of grasps is represented by a single grasp frame plus an axis of rotation (see Figure 4).

2) Action-motion plan mapping $(\xi)$ : Specifying how symbolic actions relate to motion plans is partly done in 4, which imposes start and goal configurations (Equation (3)) and continuity constraints (Equation (5)). Only motion primitives needs to be specified. From our experience, there are as many motion primitives as there are system designers. In order to keep the benchmarks accessible to a majority of planners, we only specify which joints are allowed to be actuated for a given

2 http://tampbenchmark.aass.oru.se/index.php?title=XML_format 


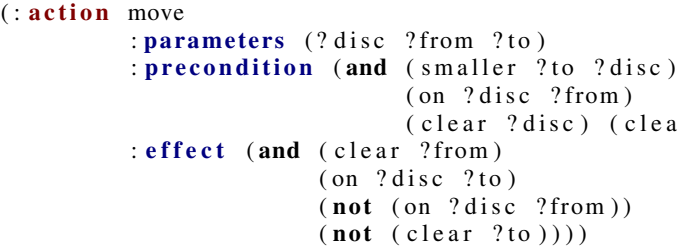

(a)

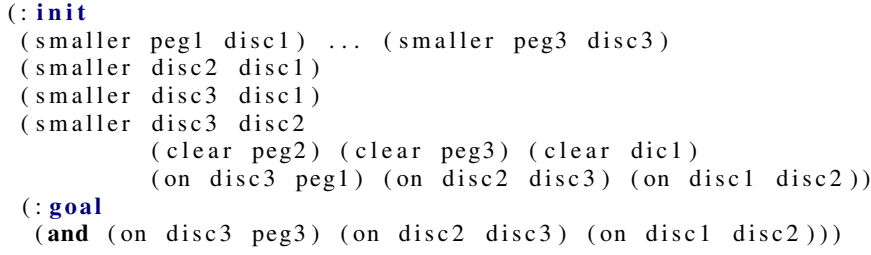

(b)

Fig. 1: Example PDDL task specification for the Towers of Hanoi problem (see subsection VI-A). (a) represents the transition function $\gamma$ of the task language (see 1) using PDDL actions with preconditions and effects. (b) represents the start and goal states of the task language using symbolic expressions.

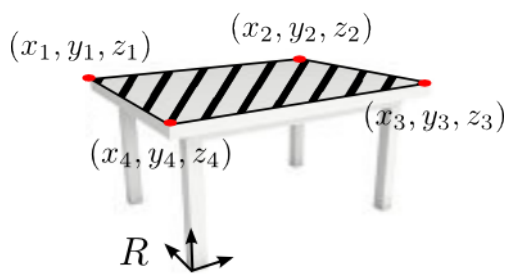

Fig. 2: Rectangular SSSP for a table represented by the list $\left(\left(x_{1}, y_{1}, z_{1}\right),\left(x_{2}, y_{2}, z_{2}\right),\left(x_{3}, y_{3}, z_{3}\right),\left(x_{4}, y_{4}, z_{4}\right)\right)$

$$
R_{1}=\left[\begin{array}{lll}
1 & 0 & 0 \\
0 & 1 & 0 \\
0 & 0 & 1
\end{array}\right]
$$$$
R_{2}=\left[\begin{array}{ccc}
1 & 0 & 0 \\
0 & -1 & 0 \\
0 & 0 & -1
\end{array}\right]
$$
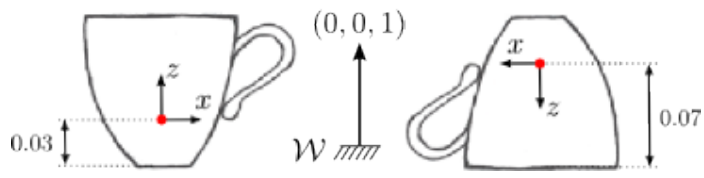

Fig. 3: Two SOPs (upright and upside down) for a cup represented by the set $\left\{\left(R_{1},(0,0,1), 0.03\right),\left(R_{2},(0,0,1), 0.07\right)\right\}$.

action. No further details about motion planning are specified, i.e., we consider the maximum number of degrees of freedom as the common ground. Note that it is perfectly valid to actuate a subset of the proposed joints. This leaves freedom for the users in the design of their motion primitives. On the other hand, it may be the case that a benchmark requires, e.g., the manipulators to be actuated separately (because the problem is more challenging that way). This would imply for some users (e.g., using only dual-arm motion planning) to implement new motion primitives to comply with the specifications. This information is given in the XML files ${ }^{4}$ aforementioned.

\section{BENCHMARKS}

This section examines five benchmark problems selected to evaluate planners based on the criteria in Table I. These criteria feature properties which make TAMP problems computationally difficult, based on scenarios from TAMP literature, experience with empirically difficult TAMP problems, and theoretical studies on difficult TAMP criteria [13], [49]. We consider the following criteria to evaluate TAMP in the fullyobservable, deterministic case:
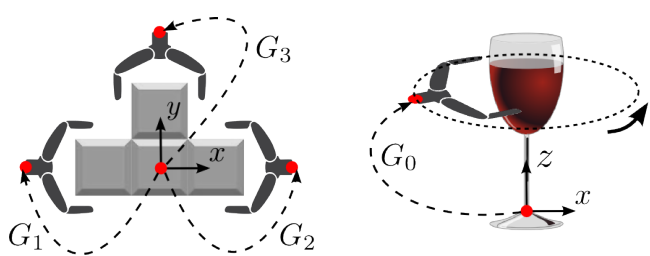

Fig. 4: A discrete grasp set : $\left\{G_{1}, G_{2}, G_{3}\right\}$ (left) and a continuous grasp set $\left(G_{o},(0,0,1)\right)$ (right).

\begin{tabular}{|l|c|c|c|c|c|}
\hline Criteria & Pb. 1 & Pb. 2 & Pb. 3 & Pb. 4 & Pb. 5 \\
\hline Infeasible task actions & $\checkmark$ & $\checkmark$ & $\checkmark$ & $\checkmark$ & $\checkmark$ \\
\hline Large task spaces & $\checkmark$ & $\checkmark$ & $\checkmark$ & & \\
\hline Motion/Task Trade-off & & $\checkmark$ & & & \\
\hline Non-monotonicity & & & & $\checkmark$ & $\checkmark$ \\
\hline Non-geometric actions & & & & & $\checkmark$ \\
\hline
\end{tabular}

TABLE I: Criteria evaluated by each benchmark problem

- Infeasible task actions: Some task actions are not possible, i.e, no corresponding motion plan exists. Possible causes of infeasibility include blocking objects and kinematic limits of the robot.

- Large task spaces: The underlying task planning problem requires substantial search effort.

- Motion/Task Trade-off: The problem can be solved with fewer steps if grasps and placements are carefully chosen.

- Non-monotonicity: Some objects need to be moved more than once for achieving the goal.

- Non-geometric actions: The problem involves actions which change discrete state $\Sigma$ but not configurations $\mathcal{C}$.

\section{A. Problem 1: Towers of Hanoi}

We extend the classic Tower of Hanoi problem to robot manipulation. The base of the robot is fixed. The rods are set in a triangular fashion and the discs are very thick, so that stacking them on a rod may temporarily create an obstacle and prevent from picking/placing discs on other rods.

This problem evaluates the large task space and infeasible task actions criteria. Although the rules are simple, the optimal solution plan for $n$ discs contains $2^{n}-1$ steps, without considering geometry (large task space). The rules of the game require certain intermediate states, many of which are 


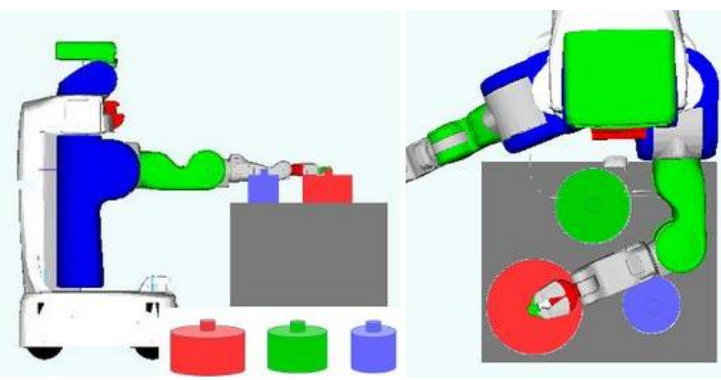

Fig. 5: The Towers of Hanoi benchmark

geometrically infeasible (infeasible task actions) if the chosen order creates occlusions.

\section{B. Problem 2: Blocks World}

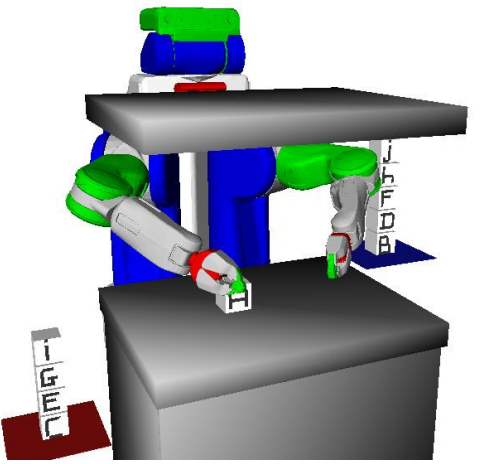

Fig. 6: The Blocks World benchmark

Another classical task planning problem, in which the goal is to stack the blocks in alphabetical order anywhere it is possible. The base is fixed, only top-grasps can be used, and hand-over is not allowed.

This problem evaluates the infeasible task actions, large task space, and motion/task trade-off criteria. An obstacle is hovering over the table, such that the grippers would collide with it if more than two blocks are stacked on the table (infeasible task actions). Both initial piles need to be unstacked somewhere and re-stacked on one of the trays. During this process, half of the blocks need to transit from one tray to another, through the table. This requires many actions (large task space). The region on the table where blocks transit has to be reachable by both arms, therefore its size is limited. One must choose between few steps and cluttered table, or many steps and uncluttered table (motion/task trade-off).

\section{Problem 3: Sort clutter}

The goal constraints are that all $N$ blue blocks must be on the left table and all $N$ green blocks must be on the right table. There are also $2 N$ red blocks acting as obstacles for reaching blue and green blocks.

This problem evaluates the infeasible task actions and large task space criteria. The close proximity of the blocks forces the planner to carefully order its operations, as well as to move red blocks out of the way without creating new occlusions

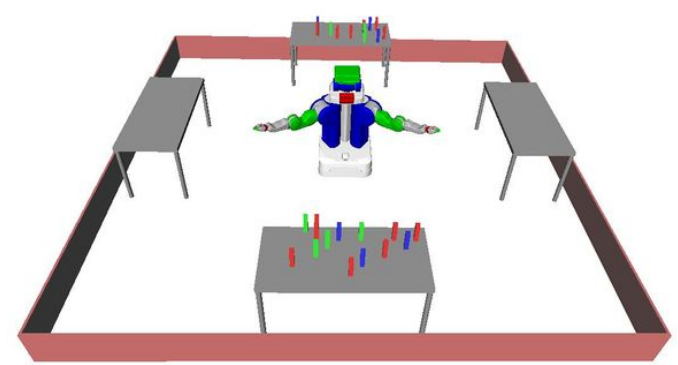

Fig. 7: The Sort Clutter benchmark

(infeasible task actions). Solving the problem requires to move many objects, sometimes multiple times (large task space).

\section{Problem 4: Non-Monotonic}

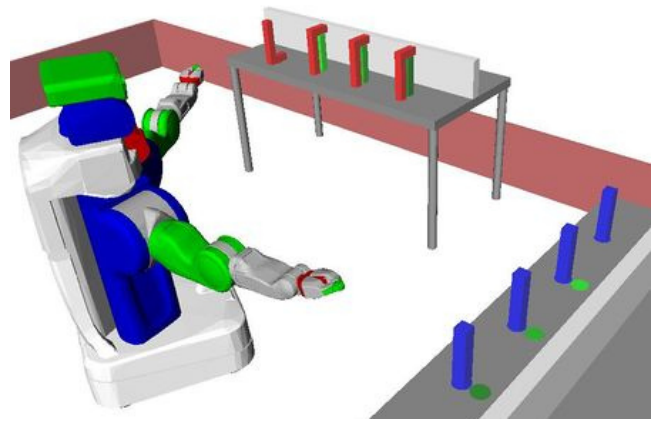

Fig. 8: The Non-Monotonic benchmark

The robot must move the green blocks from the left table to a corresponding position on the right table. In the goal state, blue and cyan blocks have to be in their initial poses.

This problem evaluates the infeasible task actions and nonmonotonicity critiera. Both the initial and goal poses are blocked by four blue and cyan blocks respectively (infeasible task actions). The goal condition of blue and cyan blocks requires to temporarily move them away and bring them back later on (non-monotonicity) in order to solve the problem.

\section{E. Problem 5: Kitchen}

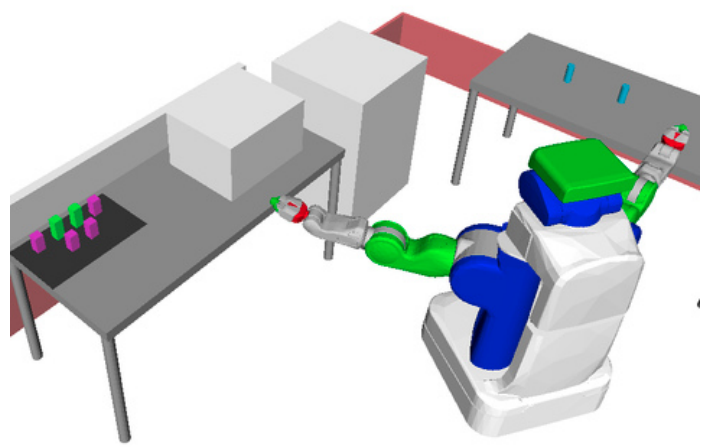

Fig. 9: The Kitchen benchmark 
The robot must "prepare a meal" by cleaning two glasses (blue blocks), cooking two cabbages (green blocks), and setting the table. Objects can be cleaned when placed on the dishwasher and cooked when placed on the microwave. An object must be cleaned before it can be cooked. Finally, the radishes (pink blocks) initially obstruct the cabbages on the shelf forcing the robot to move them. But, to maintain a tidy kitchen, the robot must also return them to their initial poses.

This problem evaluates the infeasible task actions, nonmonotonicity, and non-geometric actions criteria. A cook action is non-monotonic and both cook (via the stove) and clean (via the dishwasher) are non-geometric from the robot's perspective. Reaching target objects may also require removing blocking objects.

\section{Using The Benchmark SET}

The current benchmark set is independent of specific planners and specific robots to maximize portability to different platforms and support physical testing on available hardware. We have, however, used the PR2 as an example since it is a commonly-used robot. We anticipate that the problems will port to other robots, though some cases may require slight modification. For example, the Blocks World benchmark (see subsection VI-B) may require different placements of the trays, or a different position of the hovering obstacle, because reachability and occlusions may differ for some other robots.

Each problem is available in different scales. For instance, the Towers of Hanoi problem is proposed with 3, 4, 5, and 6 discs. For each scale, there are $N$ random variations of the initial geometric state, over which the average values of the metrics are computed.

\section{A. Files}

The proposed benchmarks are available online at the following address: http://tampbenchmark.aass.oru.se/index.php? title $=$ Problems

For each problem, we provide following data to define the task domain, motion domain, and task motion interaction:

1) A PDDL file defining the task domain

2) An archive containing all scene meshes in Wavefront OBJ format

3) An XML file describing:
a) the initial pose of all objects;
b) the initial configuration of the $\operatorname{robot}(\mathrm{s})$;
c) the initial kinematic coupling between objects;
d) which objects are movable;
e) the Surfaces Supporting Stable Placement (SSSP);
f) the Stable Object Poses (SOP);
g) Grasp Sets (optional).

\section{B. Metrics}

We propose to quantitatively compare TAMP systems based on metrics that evaluate performance of the planner itself and quality of the computed plans. Planner performance can be measured in terms of average planning times, overall success rate, or success rate within a time bound. For the current benchmarks, we propose measuring plan quality in terms of length of the computed plan, both for the number of actions in the task plan and total length of the motion plans. Multiple metrics that cover efficiency, successful instances, and plan quality will measure the trade-offs between different TAMP approaches with different focus, for example expected efficiency vs. achieving probabilistic completeness.

\section{CONCLUSION}

In this paper, we have proposed a principled approach to evaluate and compare TAMP systems. Though work in TAMP has proceeded for more than a decade, it has remained until now difficult to directly compare different planners. We have formally define the TAMP problem under full observability and determinism assumptions, presented a way to unambiguously specify TAMP problems, and proposed five benchmark problems selected to cover the known challenges of TAMP. Finally, we provide a web page (http://tampbenchmark.aass. oru.se) to download the necessary data for the benchmark problems.

Our continuing step is to organize a workshop to use and discuss this benchmark set. Based on feedback from the community, the proposed problems and specification formats may be revised and improved in several possible ways. Rather than individual problem instances, we could produce problem generators with tunable hardness parameters, based on community evaluation of the criteria in Table I. One could extend the class of problems, e.g., to partially observable, nondeterministic cases. Another possible direction is to organize a TAMP competition, which would require a common output format for plans and an automatic plan validator. We will continue development and dissemination of this benchmark set to promote direct comparison among TAMP systems.

Acknowledgments. This work is supported by the Swedish Knowledge Foundation (KKS) project "Semantic Robots" and by NSF grants IIS 1317849 and CCF-1514372 to the Kavraki Lab at Rice University, USA. This work was partially supported by the Spanish Government through the project DPI2016-80077-R. Aliakbar Akbari is supported by the Spanish Government through the grant FPI 2015.

\section{REFERENCES}

[1] N. J. Nilsson, "Shakey the robot," AI Center, SRI International, 333 Ravenswood Ave., Menlo Park, CA 94025, Tech. Rep. 323, Apr 1984.

[2] M. Stilman and J. J. Kuffner, "Navigation among movable obstacles: Real-time reasoning in complex environments," Int. Journal of $\mathrm{Hu}$ manoid Robotics (IJHR), vol. 2, no. 04, pp. 479-503, 2005.

[3] A. Krontiris and K. Bekris, "Dealing with difficult instances of object rearrangement," in Proceedings of Robotics: Science and Systems, Rome, Italy, July 2015.

[4] A. Akbari, Muhayyudin, and J. Rosell, "Task planning using physicsbased heuristics on manipulation actions," in Emerging Technologies and Factory Automation (ETFA), 2016 IEEE 21st International Conference on. IEEE, 2016, pp. 1-8.

[5] L. Karlsson, J. Bidot, F. Lagriffoul, A. Saffiotti, U. Hillenbrand, and F. Schmidt, "Combining task and path planning for a humanoid twoarm robotic system," in TAMPRA: ICAPS Workshop on Combining Task and Motion Planning for Real-World Applications, 2012.

[6] W. Vega-Brown and N. Roy, "Asymptotically optimal planning under piecewise-analytic constraints," in Workshop on the Algorithmic Foundations of Robotics, 2016. 
[7] M. Vallati, L. Chrpa, M. Grześ, T. L. McCluskey, M. Roberts, S. Sanner et al., "The 2014 international planning competition: Progress and trends," AI Magazine, vol. 36, no. 3, pp. 90-98, 2015.

[8] C. Barrett, M. Deters, L. de Moura, A. Oliveras, and A. Stump, "6 years of smt-comp," Journal of Automated Reasoning, vol. 50, no. 3, pp. 243-277, Mar 2013.

[9] H. Kitano, M. Asada, Y. Kuniyoshi, I. Noda, and E. Osawa, "Robocup: The robot world cup initiative," in Proceedings of the First International Conference on Autonomous Agents, ser. AGENTS '97. New York, NY, USA: ACM, 1997, pp. 340-347.

[10] A. Saffiotti and T. v. d. Zant, "Foreword: The impact of rockin on robotics," in RoCKIn - Benchmarking Through Robot Competitions. Rijeka: InTech, 2017, ch. 01.

[11] S. Cambon, F. Gravot, and R. Alami, "A robot task planner that merges symbolic and geometric reasoning," p. 895, 2004.

[12] C. Dornhege, P. Eyerich, T. Keller, S. Trüg, M. Brenner, and B. Nebel, "Semantic attachments for domain-independent planning systems," in Proc. of Int. Conf. on Automated Planning and Scheduling (ICAPS), 2009, pp. 114-121.

[13] M. Stilman, J.-U. Schamburek, J. Kuffner, and T. Asfour, "Manipulation planning among movable obstacles," in Robotics and Automation, 2007 IEEE International Conference on, april 2007, pp. 3327 -3332.

[14] J. Choi and E. Amir, "Combining planning and motion planning," in Proceedings of Int. Conf. on Robotics and Automation (ICRA), 2009, pp. 238-244.

[15] L. P. Kaelbling and T. Lozano-Pérez, "Hierarchical task and motion planning in the now." in Proceedings of Int. Conf. on Robotics and Automation (ICRA), 2011, pp. 1470-1477.

[16] E. Plaku, "Path planning with probabilistic roadmaps and linear temporal logic," pp. 2269-2275, 2012

[17] L. de Silva, A. K. Pandey, and R. Alami, "An interface for interleaved symbolic-geometric planning and backtracking." in IROS. IEEE, 2013, pp. 232-239.

[18] R. Dearden and C. Burbridge, "An approach for efficient planning of robotic manipulation tasks," in Proc. of Int. Conf. on Automated Planning and Scheduling (ICAPS), 2013, pp. 55-63.

[19] S. Srivastava, E. Fang, L. Riano, R. Chitnis, S. Russell, and P. Abbeel, "Combined task and motion planning through an extensible plannerindependent interface layer," in IEEE International Conference on Robotics and Automation (ICRA), 2014.

[20] C. R. Garrett, T. Lozano-Pérez, and L. P. Kaelbling, "Heuristic search for task and motion planning," in Proceedings of the Workshop on Planning and Robotics (PlanRob), 2014, pp. 148-156.

[21] E. Erdem, K. Haspalamutgil, C. Palaz, V. Patoglu, and T. Uras, "Combining high-level causal reasoning with low-level geometric reasoning and motion planning for robotic manipulation," in Proceedings of Int. Conf. on Robotics and Automation (ICRA), 2011, pp. 4575-4581.

[22] N. T. Dantam, Z. Kingston, S. Chaudhuri, and L. E. Kavraki, "Incremental task and motion planning: A constraint-based approach," in Robotics: Science and Systems, 2016.

[23] F. Lagriffoul and B. Andres, "Combining task and motion planning: A culprit detection problem," The International Journal of Robotics Research, 2016.

[24] F. Lagriffoul, "On benchmarks for combined task and motion planning," in RSS Workshop on Combined Task and Motion Planning, 2016.

[25] M. Ghallab, A. Howe, C. Knoblock, D. Mcdermott, A. Ram, M. Veloso, D. Weld, and D. Wilkins, "PDDL-The Planning Domain Definition Language," 1998.

[26] S. Edelkamp and J. Hoffmann, "Pdd12. 2: The language for the classical part of the 4th international planning competition," Technical Report 195, University of Freiburg, Tech. Rep., 2004
[27] V. Lifschitz, "Answer set programming and plan generation," Artificial Intelligence, vol. 138, no. 1, pp. 39 - 54, 2002, knowledge Representation and Logic Programming.

[28] C. Barrett, P. Fontaine, and C. Tinelli, "The SMT-LIB Standard: Version 2.5," Department of Computer Science, The University of Iowa, Tech. Rep., 2015, www.SMT-LIB.org.

[29] J. Barry, K. Hsiao, L. P. Kaelbling, and T. Lozano-Perez, "Manipulation with multiple action types," in International Symposium on Experimental Robotics, June 2012.

[30] M. Toussaint, "Logic-geometric programming: An optimization-based approach to combined task and motion planning," in (IJCAI 2015), 2015

[31] D. Nau, M. Ghallab, and P. Traverso, Automated Planning: Theory \& Practice. San Francisco, CA, USA: Morgan Kaufmann Publishers Inc., 2004.

[32] S. M. LaValle, Planning Algorithms. New York, NY, USA: Cambridge University Press, 2006.

[33] I. A. Şucan, M. Moll, and L. E. Kavraki, "The open motion planning library," Robotics \& Automation Magazine (RAM), vol. 19, no. 4, pp. 72-82, 2012.

[34] T. Dean and M. Wellman, Planning and Control. M. Kaufmann Publishers, 1991

[35] E. M. Clarke, Jr., O. Grumberg, and D. A. Peled, Model Checking. Cambridge, MA, USA: MIT Press, 1999.

[36] E. Giunchiglia, J. Lee, V. Lifschitz, N. McCain, and H. Turner, "Nonmonotonic causal theories," Artificial Intelligence, vol. 153, no. 1, pp. $49-104,2004$

[37] R. Diankov, "Automated construction of robotic manipulation programs," Ph.D. dissertation, Carnegie Mellon University, Robotics Institute, August 2010. [Online]. Available: http: //www.programmingvision.com/rosen_diankov_thesis.pdf

[38] R. Smits, H. Bruyninckx, and E. Aertbeliën, "KDL: Kinematics and dynamics library," 2011, http://www.orocos.org/kdl.

[39] I. A. Sucan and S. Chitta, "MoveIt!" 2015, http://moveit.ros.org.

[40] J. Pan, S. Chitta, and D. Manocha, "FCL: A general purpose library for collision and proximity queries," in Int. Conf. on Robotics and Automation (ICRA). IEEE, 2012, pp. 3859-3866.

[41] N. T. Dantam, Z. Kingston, S. Chaudhuri, and L. E. Kavraki, "An incremental constraint-based framework for task and motion planning," International Journal of Robotics Research, Special Issue on the 2016 Robotics: Science and Systems Conference, 2018.

[42] N. T. Dantam, S. Chaudhuri, and L. E. Kavraki, "The task motion kit," Robotics and Automation Magazine, May 2018

[43] C. R. Garrett, T. Lozano-Perez, and L. P. Kaelbling, "Backwardforward search for manipulation planning," in IEEE/RSJ International Conference on Intelligent Robots and Systems (IROS), 2015.

[44] C. Garrett, T. Lozano-Perez, and L. Kaelbling, "Sample-based methods for factored task and motion planning," in Proceedings of Robotics: Science and Systems, Cambridge, Massachusetts, July 2017.

[45] J. Rintanen, "Impact of modeling languages on the theory and practice in planning research," in $A A A I, 2015$, pp. 4052-4056.

[46] R. S. Hartenberg and J. Denavit, Kinematic synthesis of linkages. McGraw-Hill, 1964

[47] Willow Garage, "URDF XML," 2013, http://wiki.ros.org/urdf/XML.

[48] Industrial automation systems and integration - COLLADA digital asset schema specification for $3 D$ visualization of industrial data, 1st ed., ISO/PAS, 2012, http://www.iso.org/iso/catalogue_detail.htm?csnumber= 59902.

[49] K. Hauser, "The minimum constraint removal problem with three robotics applications," International Journal of Robotic Research, vol. 33, no. 1, pp. 5-17, 2014 